\title{
Application of the HICAM Camera for Imaging of Prompt Gamma Rays in Measurements of Proton Beam Range
}

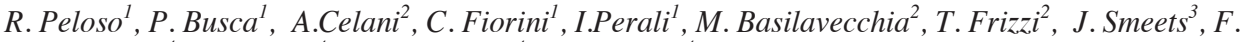 \\ Roellinghoff $^{4}$, D.Prieels ${ }^{4}$, F. Stichelbaut ${ }^{4}$, A. Benilov ${ }^{4}$ \\ ${ }^{1}$ Politecnico di Milano, Dipartimento di Elettronica e Informazione, Milano, Italy and INFN, Sezione \\ di Milano, Milano, Italy. \\ ${ }^{2}$ XGLab, Milano, Italy. \\ ${ }^{3}$ Université Libre de Bruxelles, Service de Métrologie Nucléaire, Brussels, Belgium and funded by \\ the Belgian FNRS (aspirant). \\ ${ }^{4}$ Ion Beam Applications S.A., Louvain-la-Neuve, Belgium.
}

\begin{abstract}
The HICAM gamma camera is an imaging device recently developed in the framework of a European project, based on Silicon Drift Detectors (SDDs) as photodetectors. Although originally designed for low-energy gamma-ray imaging in nuclear medicine (140 keV of $\left.{ }^{99 \mathrm{~m}} \mathrm{Tc}\right)$, in this work we attempt to use the camera, suitably modified, to image high energy prompt gamma rays ( 2 to $7 \mathrm{MeV}$ ) emitted by a target irradiated by protons. The final objective of our experiment is to assess the feasibility of proton beam range measurements by prompt gamma imaging with a slit camera, and the HICAM camera was chosen for a first prototype. Although a SDD-based camera would not be fast enough for real treatment conditions, the prototype here employed benefited from the camera modularity, compactness, high resolution and low noise. The camera here employed is composed of 25 SDDs of $1 \mathrm{~cm}^{2}$ active area each, arranged in a $5 \times 5$ format, already used in clinical and research environments with a high intrinsic spatial resolution $(\sim 1 \mathrm{~mm})$. The SDD matrix has been coupled to a LYSO crystal $(1 \mathrm{~cm}$ thickness), to improve efficiency with high-energy gammas, and has been characterized preliminarily with a ${ }^{60} \mathrm{Co}$ source. Good imaging performances have been obtained in this test. Moreover, results of a first test of the camera to detect prompt gammas emitted with a proton beam impinging on a plastic target are presented in this work.
\end{abstract}

\section{Introduction}

Particle therapy is nowadays considered an advantageous option in cancer treatment and growing research efforts are dedicated to its development. Strength of this kind of therapy, in particular proton therapy, is related to the possibility to release the maximum of the dose in the target site destroying tumoral cells and limiting otherwise the dose to healthy tissues.

For this purpose, the measurement of the proton beam range in the target is very important but although the energy of therapeutic proton beams is usually controlled with a high level of accuracy, corresponding to a sub-mm range accuracy in water, the real range of such proton beams in patients may contain uncertainties of up to $10-15 \mathrm{~mm}$ (due to uncertainties on tissue composition, internal organ motions, anatomical and physiological changes, patient positioning, etc.). 
The proposed method to improve the quality control of the treatment is based on the detection of prompt gamma rays emitted by excited nuclei during proton irradiation [1].

In fact, during a proton irradiation, the secondary emissions generate by nuclear interactions within the target include prompt gammas with energies up to $10 \mathrm{MeV}$ and previous studies have shown that the location of these prompt gammas is correlated to the position of the Bragg peak [2-3]. The development of a prompt gamma camera could be a solution which allows checking in real-time the range of a single pencil beam with a ' $\mathrm{mm}$ ' accuracy in patients [4].

Prompt gammas emitted along the proton tracks are selected with a tungsten slit collimator and detected with a $10 \mathrm{~mm}$ LYSO scintillator to obtain a reversed 1D image of the proton beam path in the target, as visible in Fig.1.

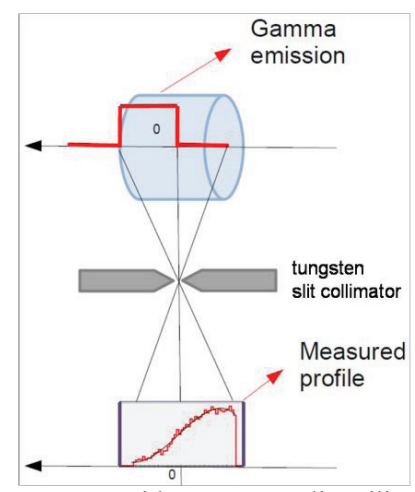

Fig. 1: Scheme of a prompt gamma camera with a tungsten slit collimator and a LYSO scintillator to detect selected prompt gamma emitted.

The challenge of this method is to detect high energy photons in an important neutron background. A dedicated concept was designed with Monte Carlo simulations and an example of the response is reported in Fig. 2 for an energy window of 3 to $7 \mathrm{MeV}$. The profile of the photons correlated to the Bragg peak is indicated in green color and in pink the contribution of neutrons which only generate an offset that does not perturb the overall correlation with the Bragg peak.

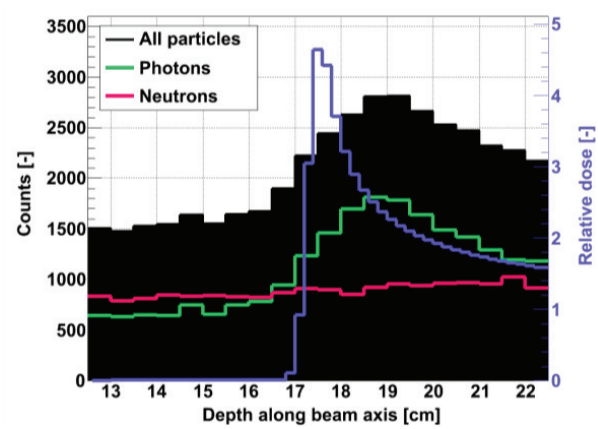

Fig. 2: . Response of a MC simulated camera in an energy window of $3-7 \mathrm{MeV}$ when sending $10^{9}$ protons of $160 \mathrm{MeV}$ on the water target. Photon and neutron count rates are about 1000 counts per $5 \mathrm{~mm}$ bin. 
To preliminarily verify the effectiveness of this concept we use the HICAM gamma camera, recently developed in the framework of the HICAM (HIgh resolution CAMera) project supported by the European Community, under coordination of Politecnico di Milano [5].

\section{The HICAM Gamma Camera}

HICAM is an Anger camera, designed for medical applications with low energy gamma sources (typ. $140 \mathrm{keV}$ of ${ }^{99 \mathrm{~m}} \mathrm{Tc}$ ), based on Silicon Drift Detector (SDD) photodetectors which are characterized by high quantum efficiency $(>80 \%)$ and low electronic noise. The basic detection unit is a monolithic array of $5 \mathrm{SDD}$ of $1 \mathrm{~cm}^{2}$ active area each. During the project two prototypes in a format $5 \mathrm{x} 5$ and $10 \mathrm{x}$ 10 were realized assembling several SDDs units, Fig. 3 shows a photograph of the 25 SDDs matrix. The photodetectors were coupled with a CsI crystal (10mm thickness). A custom designed ASIC reads and filters the signals from each SDD.

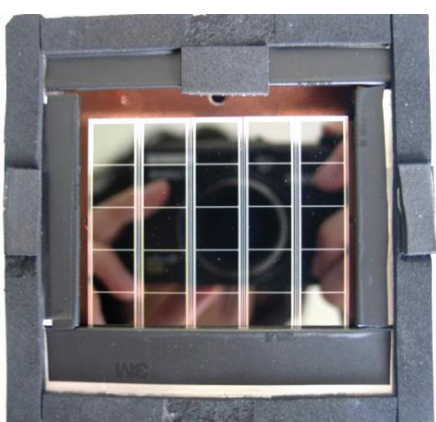

Fig. 3: Photograph of the detection module of the small HICAM prototype. The whole active area is $5 \mathrm{~cm} \times 6 \mathrm{~cm}$.

The HICAM camera is characterized by a very high intrinsic resolution $(\sim 1 \mathrm{~mm})$ [6] and has recently shown good performances both in clinical and pre-clinical applications [7].

For this work we used the small prototype with a $5 \mathrm{~cm} \mathrm{x} 6 \mathrm{~cm}$ active area. Originally developed only for low-energy gamma-ray imaging, in this work it has been suitably modified to image higher energy prompt gamma rays ( 2 to $7 \mathrm{MeV}$ ) emitted by the target irradiated by protons. The photodetectors are now coupled with a LYSO crystal of $1 \mathrm{~cm}$ thickness, a good compromise between improved detection efficiency and still satisfactory ratio between thickness/FOV. The light collection was purposely limited covering the top side and the lateral ones with black absorbing tapes to match the readout ASIC dynamic range, originally designed for $200 \mathrm{keV}$. The natural emission of Lutetium gives a significant background to the energy spectrum and suitable processing of signals has been introduced to minimize its contribution in the images.

\section{Laboratory characterization of the camera}

A first measurement session was made in Politecnico laboratory, to assess the imaging capability of the system with a ${ }^{60} \mathrm{Co}$ source $(1.17 \mathrm{MeV}, 1.33 \mathrm{MeV})$.

As schematically represented in Fig. 4 , the ${ }^{60} \mathrm{Co}$ point source was placed on the edge of a lead block (3 $\mathrm{cm}$ thickness) and moved from one boundary of the active area of the camera to the opposite one with steps of $0.5 \mathrm{~cm}$ in order to cover all the detection area in longitudinal direction. Fig.5. shows the 
reconstructed image, by means of centroid algorithm, of the irradiation of the source when placed in the middle of the camera.

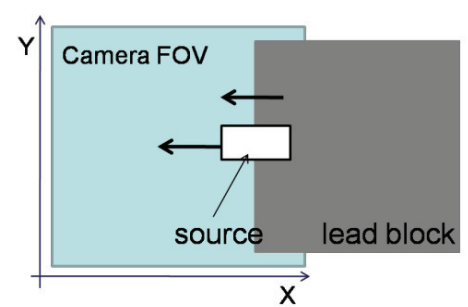

Fig. 4: $\mathrm{A}^{60} \mathrm{Co}$ point source is placed on the edge of a lead block (thickness of $3 \mathrm{~cm}$ ) and moved from one boundary of the active area to the opposite one with steps of $0.5 \mathrm{~cm}$.

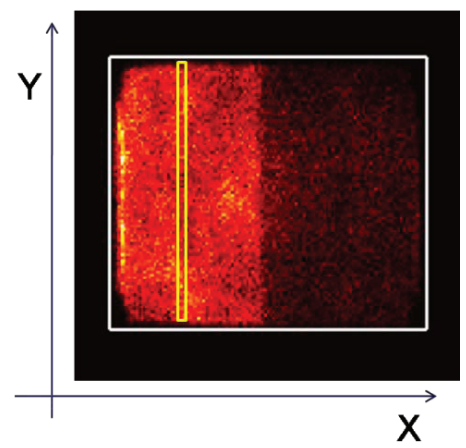

Fig. 5: Reconstructed irradiation of the ${ }^{60} \mathrm{Co}$ point source next to the middle of the camera. The centroid algorithm produces a reduction of the useful active area, pointed out by the white box.

From the figure, it is possible to notice that the irradiated region and the dark one (covered by the lead block) are separated quite sharply. The counts of the image are then integrated along the vertical axis, with $1 \mathrm{~mm}$ bin (yellow box in figure), to compute the 1D profile. All the acquired profiles of the moving source along the whole area, are overlapped and shown in Fig. 6, each one visualized only in a proper region of interest.

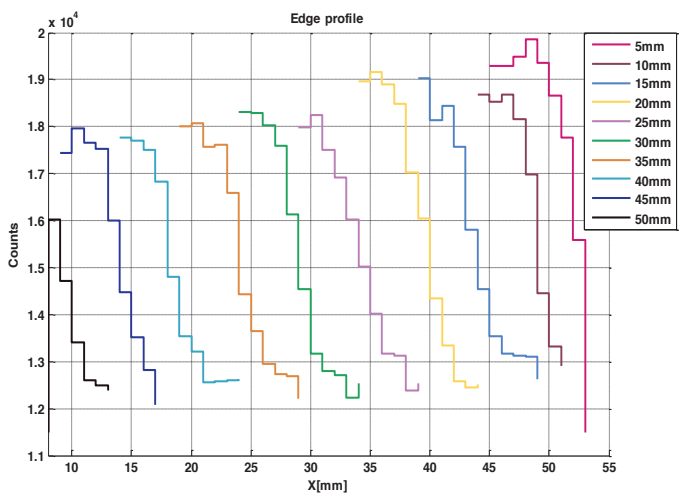

Fig. 6: Edge profiles of the source moving across the field of view with steps of $0.5 \mathrm{~cm}$. 


\section{Proton range measurements}

The first experiment with prompt gammas was carried out in the new WPE proton therapy center in Essen, Germany. The experimental set up, schematically shown in Fig. 7, consists in a proton beam impinging on a PMMA target of $20 \mathrm{~cm}$ length and $7.5 \mathrm{~cm}$ radius. A tungsten, knife-edge slit collimator, with an aperture of $6 \mathrm{~mm}$, is set at a distance of $15 \mathrm{~cm}$ to detect the prompt gammas emitted in perpendicular direction with respect to the beam one. HICAM camera is placed at the same distance of $15 \mathrm{~cm}$ from the collimator. Both the camera and the read out electronics are shielded with a lead box, $5 \mathrm{~mm}$ of thickness. A photograph of the set-up is shown in Fig. .

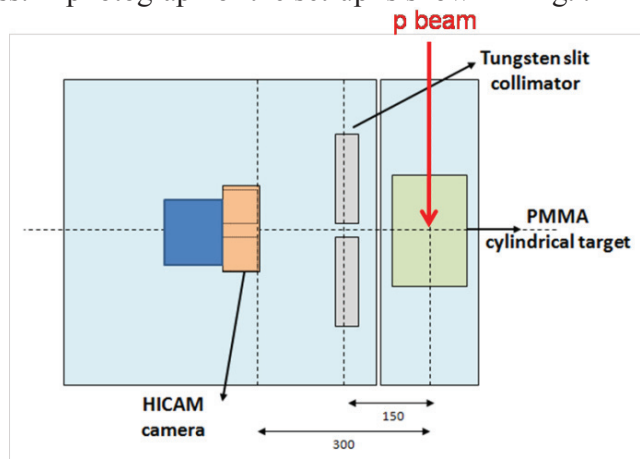

Fig. 7: Scheme of the set up for prompt gammas measurements. The slit collimator is set at a distance of $15 \mathrm{~cm}$ to detect the prompt gammas emitted in perpendicular direction with respect to the beam one. The same distance is kept between HICAM and the collimator.

In the energy spectrum of prompt gammas, as shown in the measurement of Fig., the significant component of the natural emission of Lutetium is visible but the selected energy window from 3 to 7 $\mathrm{MeV}$, set for all the following acquisitions, cuts off its contribution.

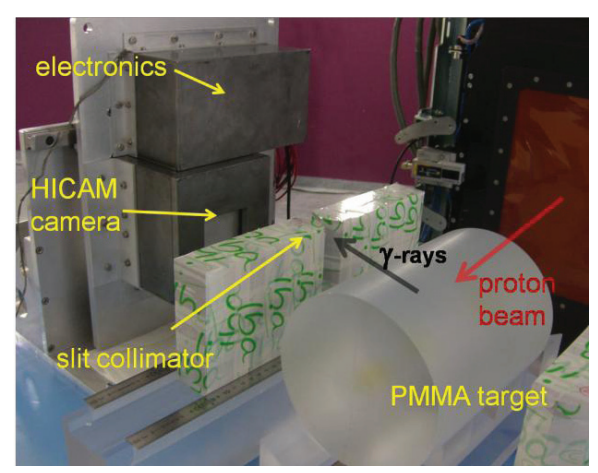

Fig. 8: Photograph of the set up used in the radiotherapy facility. The slit collimator, aperture $6 \mathrm{~mm}$, and the Hicam camera are in perpendicular direction with respect the proton beam one. 


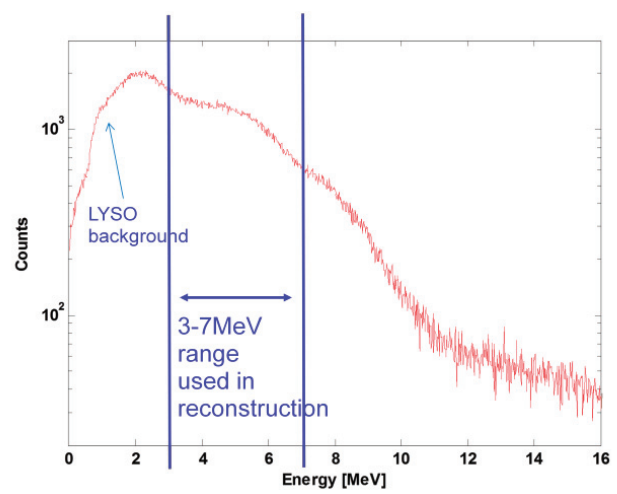

Fig. 9: Energy spectrum of prompt gammas. The energy window of 3-7 MeV is selected for the reconstruction.

We first present an acquisition ( 5 min long) in which the proton beam was set at an energy of 160 $\mathrm{MeV}$.

In the default configuration of the setup, the dose peak is aligned with the collimator slit and the center of the camera FOV (see Fig. 7). The HICAM camera has also a mobile support that allows to place the camera in two other positions at distances of $4 \mathrm{~cm}$ toward right and left. The same acquisition was thus repeated with the camera in these two positions and the reconstructed profiles are overlapped in the same graph. This allows to better image the whole profile despite the limited FOV of HICAM.

The profiles obtained from the three acquisitions are shown in Fig. 10a. Another set of three measurements was carried out substituting the slit collimator with a tungsten block, $4 \mathrm{~cm}$ of thickness, in order to acquire signal due to neutrons and scattered gamma-rays and the result is reported in Fig. 10b. This profile is rather flat and represents a constant contribution that could be subtracted from the previous acquisition. The resulting profile is presented in Fig. 10c and is in good agreement with simulations.

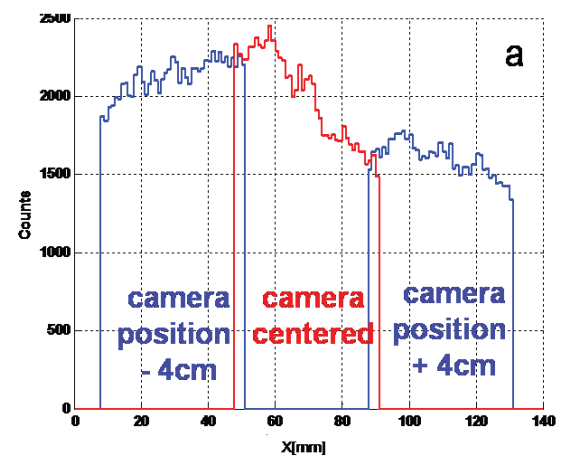



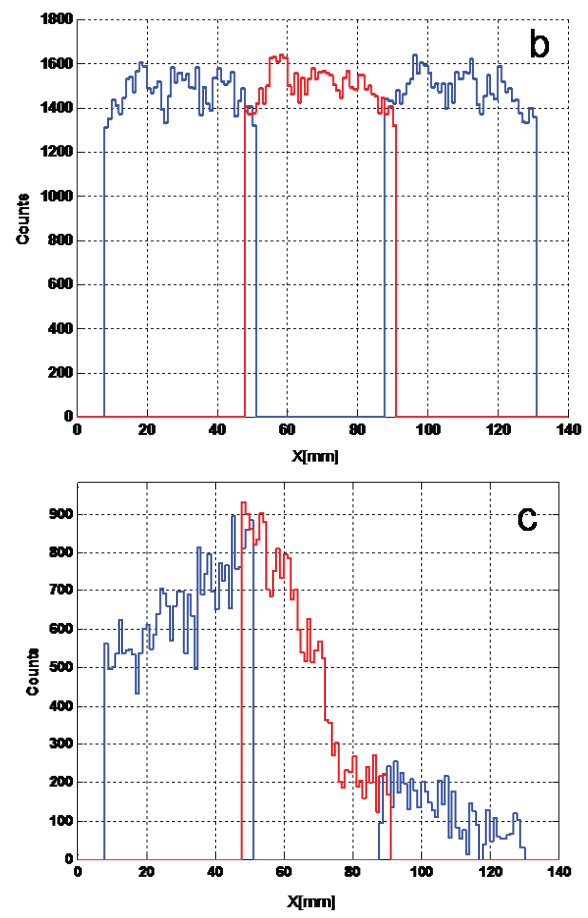

Fig. 10: (a) Profiles of three overlapped acquisitions, each of 5 min, with HICAM moving with respect to the collimator aperture; (b) contribution from neutrons and scattered photons acquired interposing a tungsten block; (c) profile obtained subtracting the last profile from the first one, that can be attributed to prompt gamma photons.

With the camera fixed in the central position another set of acquisitions was carried out changing the position of the PMMA target, with beam energy of $100 \mathrm{MeV}$. From the central position, the target was moved with steps of different length, from $1 \mathrm{~mm}$ to $10 \mathrm{~mm}$, toward both the right and the left directions, in order to evaluate the capability of the system to track also small shifts. Acquisitions time was of 5 min each.

The fits of the measured profiles are reported in Fig. 11. All the fitted profiles are well defined and separated and even the difference between $1 \mathrm{~mm}$ shifts of the target could be clearly identified by eye.

To preliminarily evaluate the accuracy of the measured shift, a reference value $\mathrm{x}_{0}$ was derived from the intersection between the curve acquired in the central position and the vertical axis in the middle of the camera FOV $(30 \mathrm{~mm})$. For each profile the distance with respect to this point is evaluated along the horizontal axis (Fig. 12). The accuracy of this measurement is preliminarily evaluated as the difference between the measured value and the expected one, as shown in Fig. 12, and it turns out to be in the ' $m$ m' range. 


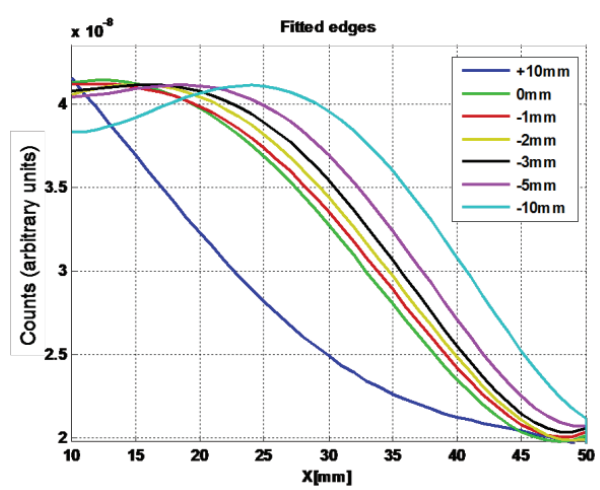

Fig. 11: Fitted profiles of the acquisitions with the moving target. Each acquisition is $5 \mathrm{~min}$ long and corresponds to $7 \cdot 10^{10}$ absorbed protons.

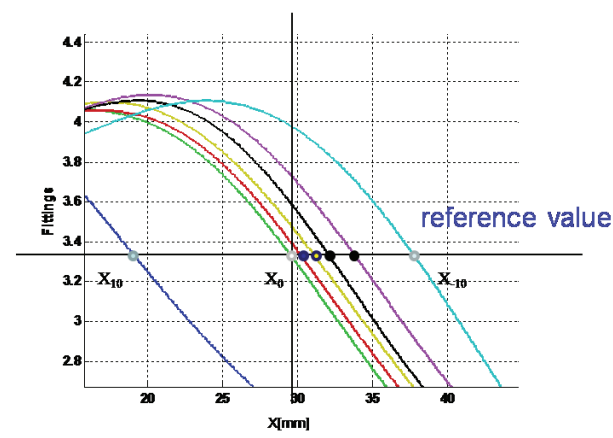

Fig. 12: The reference value $x_{0}$ was defined from the intersection between the profile of the base position and the vertical axis in the middle of the camera FOV $(30 \mathrm{~mm})$. For each profile the distance with respect to this point is evaluated along a horizontal axis.

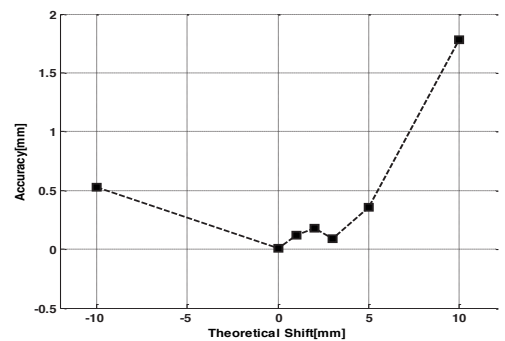

Fig. 13: Plot of the difference between exact range shift and measured shift.

Measurements were then extended to $230 \mathrm{MeV}$ to cover the full clinical range of beam energies.The profiles obtained from the three acquisitions, after the subtraction of the contribute due to neutrons and scattered gamma-rays, are shown in Fig. 10. 


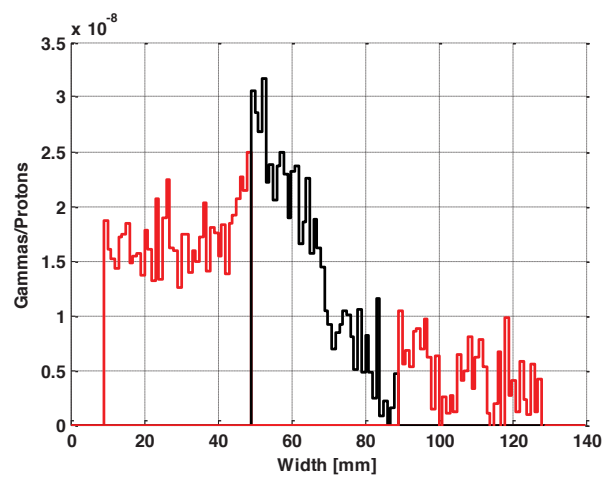

Fig. 14: Profiles of three overlapped acquisitions at $230 \mathrm{MeV}$, each of $450 \mathrm{sec}$, with HICAM moving with respect to the collimator aperture. The contribution from neutrons and scattered photons was subtracted.

Also in this case, the target was moved from the central position with steps of lengths from $1 \mathrm{~mm}$ to $10 \mathrm{~mm}$, toward both the right and the left directions. Acquisitions time was of $450 \mathrm{sec}$ each. The fits of the measured profiles are reported in Fig. 15.

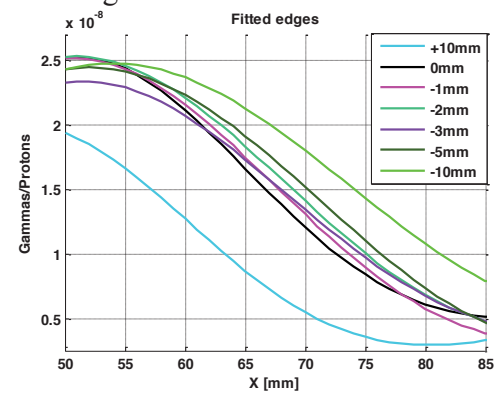

Fig. 15: Fitted profiles of the acquisitions with the moving target. Each acquisition is $450 \mathrm{sec}$ long and corresponds to $4 \cdot 10^{10}$ absorbed protons.

\section{Conclusions}

Measurements of prompt gammas correlated to proton range were successfully made using the HICAM gamma camera. Although not optimized for 1 to $10 \mathrm{MeV}$ energy range (low efficiency, low scintillator light collection) a satisfactory accuracy in the 'mm' range was first measured.

Although suitable for the measurements performed in this work, the SDD-based camera shows intrinsic limitations in terms of speed, which make it unsuited to patients treatments. The camera, however, thanks to its high intrinsic spatial resolution, compactness and versatility has allowed demonstrating the concept of slit camera for prompt gamma detection in proton therapy.

\section{Acknowledgement}

The authors thank D. Clivio, P.Compagnoni, S. Masci and S. Incorvaia for their support. 


\section{References}

[1] F. Stichelbaut, Y. Jongen," Verification of the proton beam position in the patient by the detection of prompt gamma-rays emission", 39th PTOCG meeting, San Francisco (2003).

[2] C.-H. Min, C.H. Kim, M.-Y. Youn, J.-W. Kim, "Prompt gamma measurements for locating the dose falloff region in the proton therapy", Applied Physics Letters 89: 183517 (2006).

[3] D. Kim, H. Yim, J.-W. Kim, "Pinhole camera measurements of prompt gamma-rays for detection of beam range variation in proton therapy", Journal of the Korean Physical Society 55(4): 16731676 (2009).

[4] D.Prieels, J.Smeets, F.Stichelbaut, A.Benilov, J.C.Dehaes, A.Dubus, F.Roellinghoff, "Towards a practical solution for real-time measurement of the proton beam range in patients", 50th PTCOG meeting, Philadelphia (2011).

[5] www.hi-cam.org.

[6] R.Peloso, P.Busca, C.Fiorini, A.Abba, A.Geraci, A.Manenti, A. Longoni, G.Padovini, C.Bianchi, G.L.Poli, K.Erlandsson, B.F.Hutton, P.Lechner, H.Soltau, L.Strüder, A.Pedretti, P.Van Mullekom, "The HICAM Gamma Camera" Nuclear Science Symposium Conference Record (NSS/MIC), 2010 IEEE , ISBN \# 978-1-4244-9104-9, 4 Pages.

[7] P.Busca, R.Peloso, C.Fiorini, A.Gola, A.Abba, K.Erlandsson, B.F.Hutton, C.Bianchi, G.L.Poli, U.Guerra, G.Virotta, L.Ottobrini, C.Martelli, G.Lucignani, A.Pedretti, P.Van Mullekom, S.Incorvaia, F.Perotti, "Applications of the HICAM Gamma Camera", Nuclear Science Symposium Conference Record (NSS/MIC), 2010 IEEE , ISBN \# 978-1-4244-9104-9, 4 Pages. 